\title{
ESTRATEGIAS DE ESPECTACULARIZACIÓN EN LAS TERTULIAS POLITICAS TELEVISIVAS. CASO DE LA COBERTURA DE LAS ELECCIONES MUNICIPALES DE BARCELONA DE 2015
}

\author{
Spectacularization strategies in the TV political talk \\ shows. The case of the Barcelona local election \\ campaign (2015)
}

Marta Montagut y Nereida Carrillo

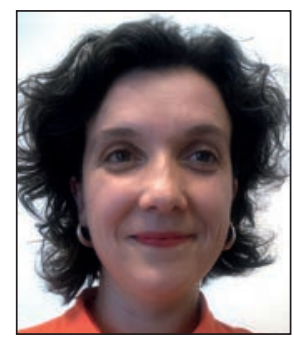

Marta Montagut es profesora lectora del Departamento de Estudios de Comunicación de la Universidad Rovira y Virgili. Miembro del grupo de investigación Asterisc, ha publicado diversos artículos sobre comunicación política, framing y radio en revistas como Journalism studies o Radio journal. Actualmente está trabajando en el análisis crítico de la metáfora en los discursos políticos y en la memoria y postmemoria en los productos mediáticos y culturales.

http://orcid.org/0000-0002-0270-9983

\author{
Universidad Rovira i Virgili. Facultad de Letras \\ Departamento de Estudios de Comunicación \\ Campus Catalunya. Avinguda Catalunya 33-35. 43003 Tarragona, España \\ marta.montagut@urv.cat
}

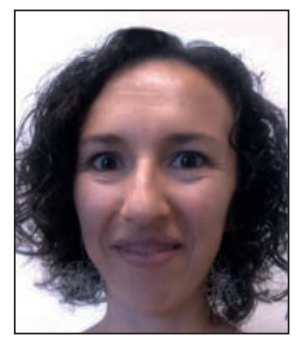

Nereida Carrillo es doctora en Comunicación por la Universitat Autònoma de Barcelona (UAB), periodista, docente e investigadora. Ha trabajado en la Cadena SER y la web de noticias de TV3 y en la actualidad colabora con el diario ARA y la revista Capçalera del Colegio de Periodistas de Cataluña. Ha impartido docencia en la Universitat Pompeu Fabra, UAB, Universidad Rovira y Virgili y Universitat Oberta de Catalunya. Se doctoró cum laude y con premio extraordinario con una tesis sobre el infoentretenimiento en las campañas electorales de 2008 y 2010 . Fruto de la investigación, publicó El periodismo volátil (editorial UOC).

http://orcid.org/0000-0002-9549-7966

Universidad Autònoma de Barcelona Facultad de Ciencias de la Comunicación Edificio I, calle de les Vinyes, s/n, 08290 Cerdanyola del Vallès (Barcelona), España nereida.carrillo@uab.cat

\section{Resumen}

La tertulia política se considera un género opinativo coral sin una codificación clara. Su amplia presencia en el ecosistema mediático español y sus niveles de audiencia son factores que apuntan al papel central de estos espacios en las programaciones convencionales de televisión y radio. La flexibilidad del género en la construcción del discurso político y la aparente pluralidad ideológica de sus actores hacen de la tertulia un espacio idóneo para detectar elementos de espectacularización de los contenidos políticos como la personalización, la dramatización, la entronización de la anécdota o la argumentación emocional. Se analizan las tertulias televisivas diarias de dos televisiones de ámbito autonómico -Televisió de Catalunya (TV3) y $8 T V$ - y una local, Barcelona TV, durante la campaña electoral municipal de Barcelona (2015). El objetivo es detectar la presencia y articulación de elementos de espectacularización mediática.

\section{Palabras clave}

Espectacularización; Infoentretenimiento; Tertulias políticas; Mediatización; Campañas electorales; Elecciones; Televisión; TV3; 8TV; BTV.

\section{Abstract}

Political talk shows -called "tertulias" (media gatherings) in Spain- are a dialogical opinion genre without a clear codification. Their wide-ranging presence and large audiences in the Spanish media system are the reason for the central role of this 
type of programming in radio and television. These political media gatherings allow for a flexible political discourse process. However, the selection of "opinion-makers" shows a "false" internal plurality that facilitates the spectacularization of political content through the political personification and dramatization of personal anecdotes which heighten the emotional register. In this article two daily regional TV political talk shows -one public (Televisió de Catalunya, TV3) and one private (8TV)- and one public local broadcaster (Barcelona Televisió, BTV) are analyzed to detect elements of spectacularization from the campaigns of Barcelona's local elections (2015).

\section{Keywords}

Spectacularization; Infotaintment; Political gathering; Mediatization; Election campaigns; Elections; Television; TV3; 8TV; BTV.

Montagut, Marta; Carrillo, Nereida (2017). “Estrategias de espectacularización en las tertulias políticas televisivas. Caso de la cobertura de las elecciones municipales de Barcelona de 2015”. El profesional de la información, v. 26, n. 4, pp. 621-629.

https://doi.org/10.3145/epi.2017.jul.06

\section{Introducción}

El fenómeno de la espectacularización mediática de los contenidos políticos responde a una dinámica de "mediatización" de la política. La mediatización se ha definido como el proceso que ubica a los medios de comunicación como actores sociales clave a través de los cuales se construye el discurso político, con lógicas productivas que imponen su lenguaje y ritmos a la actividad política (Castelló, 2012), generando una "negociación continua" entre los dos principales actores de la esfera pública -periodistas y políticos- en la construcción del discurso público (Casero-Ripollés, 2008, p. 122).

Las cadenas televisivas generalistas utilizan estrategias de espectacularización como: personalización, construcción dramática de los actores políticos, entronización de la anécdota o búsqueda del impacto emocional en las formas

En España, esta negociación responde a una dinámica sistémica -lo que Hallin y Mancini (2004) denominan el modelo mediático de pluralismo polarizado- según el cual los medios de comunicación en el Sur de Europa están fuertemente influenciados por los partidos políticos. En este contexto, la tertulia aparece como un formato opinativo estratégico que permite un acceso amplio de los discursos políticos a la antena y una capacidad de construcción discursiva espectacularizada de los contenidos políticos (León-Gross; Gómez-Calderón, 2011; Castelló; Montagut, 2011). Las cadenas televisivas generalistas compiten en el mercado de la tertulia política y utilizan estrategias propias de la espectacularización como:

- personalización;

- construcción dramática de los actores políticos;

- entronización de la anécdota sobre la historia valiosa; o

- búsqueda del impacto emocional en las formas.

Esta espectacularización del contenido político se hace evidente durante las campañas electorales (Bennet, 1992; Carrillo, 2012). Bajo estos parámetros, seleccionamos las tertulias televisivas diarias de TV3, 8TV y BTV durante la campaña de las elecciones municipales de Barcelona de 2015, para detectar si existen estos elementos y cómo se articulan para construir desde los medios la actualidad política.

\section{Encuadre de la espectacularización. Elementos del entretenimiento en el discurso político}

Consideramos el infoentretenimiento o infotainment como una tendencia que implica una hibridación entre las formas del género informativo con las características propias del entretenimiento (Brants, 1998; Jones, 2005; Kellner, 2003).

El entretenimiento no sólo impregna la información, sino que también se entremezcla con la política, creando lo que Thussu (2007) Ilama politainment. En este sentido, por lo que respecta a la información política y a las noticias políticas comentadas en las tertulias, podemos hablar de infotainment del politainment (Carrillo, 2012). Podemos enmarcar el infoentretenimiento como una manifestación mediática de una tendencia más amplia, la espectacularización, que afecta a otros espacios de la vida pública (Debord, 1992).

La academia y el mundo profesional están lejos de un consenso respecto a la definición y los efectos de la espectacularización de la información en general y de la información política en particular. Algunos autores (Postman, 1990; Debord, 1992) consideran este fenómeno como una degradación de la información y una estrategia para la pasividad y resignación de los ciudadanos respecto al mundo que les rodea. Otros instan a valorar el significado ciudadano del entretenimiento (O'Donnell, 2007), a reconocer que un modelo determinado puede ser empoderador (Bennett, 1992), o a valorar que capta públicos alejados de la información política (Van-Zoonen, 2005; Thussu, 2007) y aumenta el conocimiento de la política por parte de los ciudadanos (Ferré-Pavía; Sintes-Olivella; Gayà-Morlà, 2012).

En este estudio nos proponemos ver con qué elementos se presenta la espectacularización en las tertulias políticas tan- 
to en los temas como en la descripción de los actores y el lenguaje oral y televisivo.

En los temas, la cobertura mediática espectacularizada implica una dramatización de los asuntos públicos: la realidad se construye como un relato, como un compendio de anécdotas (Salmon, 2008) que pierden significado ciudadano. La estrategia y lo morboso eclipsan las propuestas, las políticas y el análisis, como demostró el estudio de Carrillo (2012) sobre la cobertura mediática de las elecciones catalanas de 2008 y las generales de 2010. En ambos casos, sólo en el $12 \%$ de las noticias el tema principal giraba alrededor de una propuesta, mientras que las piezas que versaban sobre la estrategia de la campaña o la historia personal de los candidatos superaban el $80 \%$. Como afirma Aira $(2009$, p. 9) la política de hoy implica "la sumisión del qué al cómo".

Los actores de las noticias tratadas en la tertulia se describen como héroes y antihéroes (Bennett, 1992; Edelman, 1988), en unos discursos que asimilan los métodos de la ficción a la vez que se alejan de los cometidos periodísticos de descripciones completas, críticas y plurales. A esta imagen creada de los políticos como ganadores y perdedores contribuyen las encuestas de valoración política y los sondeos electorales, que convierten la información en una competición o, como se ha llamado por influencia del mundo anglosajón, en una horse race (Maarek, 1997). Este tipo de cobertura es lo que Cappella y Jamieson (1997) denominan strategy coverage, entendida como una forma de narrar la política en general, y las campañas políticas en particular, que apunta a la simplificación argumental, la personalización del político y la metapolítica como tema central.

Los tertulianos-estrella, que provocan tanto en el fondo como en las formas, se prefieren a los instruidos en la materia

La espectacularización también opera cambios en las formas y el lenguaje: tanto la realización televisiva como el lenguaje corporal y el léxico empleado buscan emocionar más que describir con rigor. El discurso del miedo se conjuga con figuras literarias como las metáforas, pero también con léxico propagandístico, bélico y emocional. Como plantea Carrillo (2013, p. 65), el lenguaje se apoya en palabras "que esconden, que edulcoran, que colorean, que exageran, que disimulan". Además los ataques verbales se entremezclan con aspavientos: los tertulianos "actúan" en el sentido más teatral del término.

La espectacularización en el lenguaje verbal y no verbal de los tertulianos se acompaña de un lenguaje televisivo que busca también lo dramático. Ortells-Badenes (2015) reinterpreta y actualiza los rasgos estilísticos del infoentretenimiento en televisión estudiados primero por Früh y Wirth (1997) y más tarde por otros (Berrocal-Gonzalo et al., 2014). Aunque el análisis se centra en magazines de actualidad, algunas características son aplicables a la tertulia, como el uso de travellings para crear dinamismo, la elección de planos picados y contrapicados, los planos cortos para provocar alguna sensación en el espectador o la inserción de música y efectos de montaje en los vídeos que introducen los temas en la tertulia.

La dramatización genera la creación de una especie de star system a través del fenómeno de la personalización. Ésta atañe tanto a los políticos y otros protagonistas de la vida pública como a los tertulianos que en la actualidad frecuentan los principales programas de radio y televisión. En el primer caso, la personalización se da cuando la faceta privada del personaje trasciende a su vertiente pública. En los políticos, esta personalización descansa sobre tres pilares (Van-Santen; Van-Zoonen, 2010):
- referencias a su competencia individual;
- importancia de episodios de su vida privada;
- interés por sus emociones y opiniones.

De la misma manera, podemos afirmar que la personalización en los tertulianos se da cuando el perfil personal se antepone a su cualidad de experto. Los tertulianos-estrella, que provocan tanto en el fondo como en las formas, se prefieren a los instruidos en la materia que se alejan de las formas de la espectacularización. Así lo prueba Abejón-Mendoza en su estudio de tertulias de 13 medios de comunicación $y$ en el que concluye que:

"la credibilidad de los opinantes ha dejado de ser un valor y un motivo para buscarles. Se les busca, sobre todo, por su capacidad para generar controversia y discusión" (Abejón-Mendoza, 2013, p. 97).

Aquellos tertulianos que consiguen esa personalización, esa creación dramática de un personaje, entran en un "ecosistema" comunicativo de tertulianos que "hacen el circuito" de medios (Castelló; Montagut, 2011; Abejón-Mendoza, 2013, p. 100) y que responden a una clara uniformidad de perfiles sociales, genéricos y profesionales (Pont-Sorribes et al., 2016; Abejón-Mendoza, 2013; Altarriba-Piguillem, 2013). Además de los tertulianos-estrella, con frecuencia moderan las tertulias periodistas-estrella, que no se limitan a ordenar el debate, sino que también intervienen.

Todos estos factores nos llevan a considerar la tertulia como un género que alberga formas de espectacularización obvias en sus estrategias de construcción del discurso político y mediático, tanto a nivel de contenido como a nivel formal.

\section{La tertulia: importancia estratégica para los partidos políticos}

La tertulia no tiene una delimitación genérica clara ya que puede tener elementos de géneros clásicos de la información y la opinión (Rico-Jerez, 2014; León-Gross; Gómez-Calderón, 2011). Aun así podríamos considerarla como un género opinativo dialógico (Rico-Jerez, 2014, p. 874-875). Su presencia constante en las programaciones televisivas y radiofónicas tiene diversas razones (Toral-Madariaga, 1998; Sánchez-Serrano, 2006; León-Gross; Gómez-Calderón, 2011; Rico-Jerez, 2014; Pont-Sorribes et al., 2016):

- rehúye los límites temporales y de contenido de los géneros puramente informativos;

- permite construcciones discursivas más libres a nivel de selección léxica y de uso de figuras retóricas;

- permite la pluralidad opinativa política a través de diver- 
sos actores que no son políticos en activo sino periodistas, profesionales liberales o incluso opinadores profesionales, hecho que legitima aparentemente la neutralidad del medio;

- a nivel productivo, llenan un gran espacio de tiempo a bajo coste con buenos resultados de audiencia.

Otros autores apuntan a otros factores como la excesiva homogeneidad del sistema audiovisual español (Gutiérrez-García; Huertas-Bailén, 2004) o la tradición cultural y mediática del país (Blasco-Duatis, 2013). La alta competitividad televisiva de los años 90 entre cadenas con programaciones generalistas muy similares, genera una tendencia creciente a la espectacularización en el tratamiento de los contenidos políticos como estrategia para atraer al target potencial (Cebrián; Berrocal-Gonzalo, 2013).

La presencia continua de tertulias políticas indica la importancia de este tipo de espacios tanto para la audiencia como para la clase política

La presencia continua de tertulias políticas indica la importancia de este tipo de espacios tanto para la audiencia como para la clase política. Castelló y Montagut (2011) detectan que los jefes de prensa de los partidos políticos ponen especial énfasis en monitorizar a tertulianos y contenidos para saber con exactitud quién decía qué y en qué medio. Según León-Gross y Gómez-Calderón (2011, p. 69), uno de los motivos de la utilidad política de la tertulia es su capacidad "de fijar el temario dominante y sus encuadres". Esto, como indican Pont et al. (2016) en su análisis del pluralismo ideológico en las tertulias radiofónicas en el contexto político de Catalunya en 2014, indica la capacidad de la tertulia para sintonizar u operar como espejo de la opinión pública, aunque con elementos de politización encubierta de los tertulianos y un exceso de fuentes políticas en detrimento de la figura del experto. La tertulia es un género flexible donde se mezcla información, interpretación y opinión y donde no hay necesidad ni de una estructura rigurosa ni de una argumentación trabajada. De esta manera, "a menudo prevalece el ingenio sobre la argumentación sólida y estructurada" (León-Gross; Gómez-Calderón, 2011, p. 68).

\section{Metodología}

El estudio se centra en las tertulias de 3 televisiones de ámbito autonómico y local durante las dos semanas de la campaña electoral para las elecciones al Ayuntamiento de Barcelona (8 a 22 de mayo de 2015). Se escogieron:

- tertulia política del programa Els matins de TV3 como espacio de referencia del ecosistema público catalán;

- tertulia política vespertina del programa 8 al dia de la televisión privada 8TV (Grup Godó), la única cadena televisiva autonómica privada;

- para captar la dinámica de información y opinión de proximidad, escogimos la televisión local pública Barcelona Televisió (BTV) y su tertulia política de referencia, La rambla, emitida en franja nocturna.
La muestra comprende un total de 29 tertulias: 10 de Els matins de TV3, 11 de 8 al dia y 8 de La rambla. Los dos primeros espacios se emiten de lunes a viernes, mientras que el último lo hizo de lunes a jueves. En el caso de TV3, el día 12 de mayo no hubo tertulia puesto que se emitió un debate especial con los candidatos. De todas estas tertulias, se analizan sólo aquellos fragmentos que hacen referencia explícita a las elecciones municipales en Barcelona. Para cada tertulia se abre una ficha de análisis que comprende tres partes:

a) Una parte general donde se recoge información identificativa;

b) El perfil de los tertulianos, teniendo en cuenta especialmente su profesión y trayectoria. Se dividen los tertulianos en una doble clasificación según su función dentro de la tertulia -especializados/generalistas- según los parámetros teóricos expuestos;

c) Indicadores de la espectacularización: se analizan en primer lugar los temas abordados: si son propuestas o cuestiones relativas a la metapolítica, como la campaña electoral, las encuestas o las estrategias de los partidos. Otro factor de detección son los tipos de argumentación utilizados. Basándonos en las definiciones de la espectacularización antes expuestas y que entienden esta tendencia como una preponderancia de lo ligero ante lo sólido, optamos por clasificar en:

- argumentos basados en cifras, análisis de contexto, etc.;

- argumentos basados en falacias, tópicos, rumores, impresiones o sensaciones.

Esta categorización permite captar el nivel de profundidad y especialización con que son tratados los temas en la tertulia.

Otro de los elementos de la ficha de análisis es el uso de los recursos narrativos de la strategy coverage como elementos de detección de la simplificación narrativa asociada al fenómeno de la espectacularización de la información política. Siguiendo las características indicadas por Capella y Jamieson (1997), recogemos en la ficha de análisis el uso de:

- léxico bélico o relacionado con la competición;

- recurso de la personalización;

- tendencia a la dramatización argumental (storytelling);

- argumentos metapolíticos;

- uso sistemático de las encuestas como base de la defensa o ataque respecto a determinados temas.

La tertulia es un género flexible donde se mezcla información, interpretación y opinión, y donde no hay necesidad ni de una estructura rigurosa ni de una argumentación trabajada

Finalmente, se estudiaron los rasgos del infoentretenimiento detectables en el lenguaje oral, no verbal y el televisivo. Estudiamos si hay interacción o interrupción entre los tertulianos, si el tono resulta tranquilo o enfático e incluso exaltado, así como la disposición del plató, la realización televisiva, los movimientos de cámara o el uso de la música y otros efectos. 


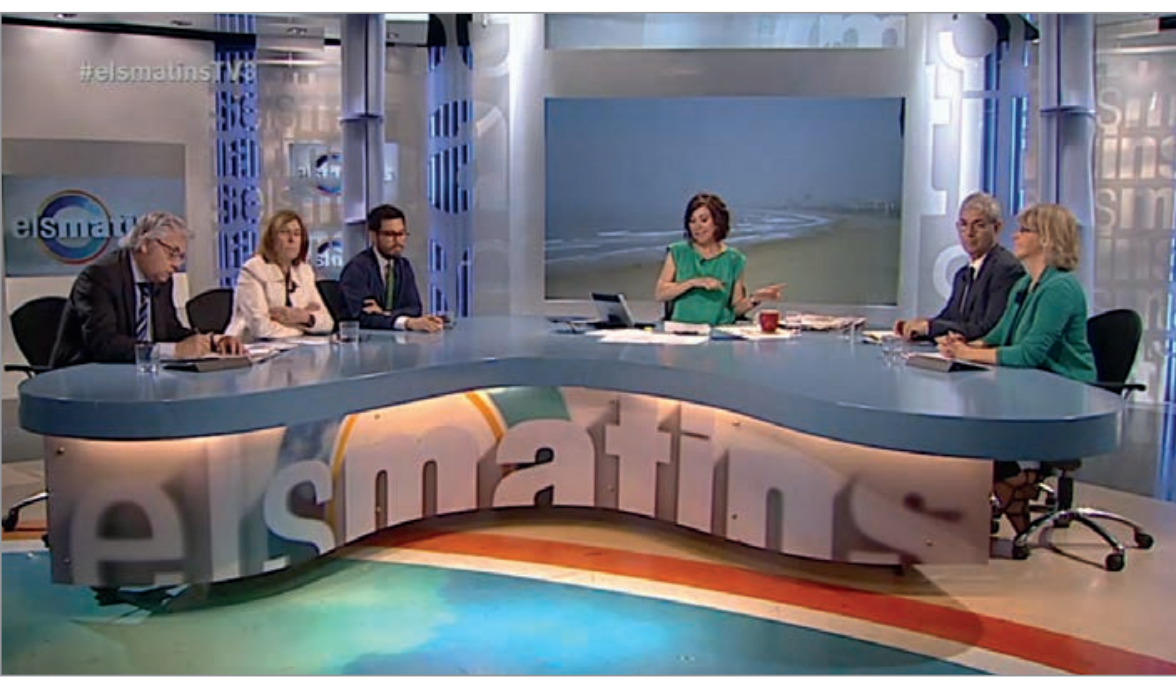

Tertulia Els matins (Las mañanas), de la cadena autonómica pública TV3

se comenta el plan urbanístico para la Avenida Diagonal o el modelo turístico de la ciudad, especialmente en la televisión local BTV. En ambos casos los temas en términos de conflicto y de personalización de los dos principales candidatos, Ada Colau, candidata por BCN en Comú (o directamente "La Colau"), y el entonces alcalde Xavier Trías, de CiU.

Tanto en las propuestas como en otros temas, destaca que aunque en la mayoría de las tertulias analizadas se representan las diversas posturas, este interés no responde tanto a una intención de pluralidad ideológica

\section{Resultados}

El perfil de los tertulianos coincide con los resultados de estudios similares: se detecta una presencia mayoritaria de hombres por encima de los 40 años, en su mayoría periodistas con un perfil generalista, aunque también aparecen de forma puntual perfiles de expertos: historiadores, economistas, abogados o filósofos.

Se apuesta por periodistas y expertos estrella, que se comunican desde una ideología marcada y con formas espectacularizadas. Cabe destacar también la aparición de expolíticos (Montserrat Nebrera o Manuel Milián Mestre) y de una abogada (Andrea Levy) que, después de su experiencia como tertuliana, pasó a tener un cargo en el Partido Popular (PP), un indicador de que los partidos políticos a veces utilizan las tertulias como campo de entrenamiento.

En los temas abordados conviene recalcar en primer lugar la preponderancia de debates de actualidad externos a la dinámica electoral: los comicios no colonizan las tertulias en ningún caso. Respecto a los temas directamente relacionados con las votaciones, los resultados coinciden con la investigación de Carrillo (2012): la presencia de las propuestas es minoritaria e impregnan el debate elementos de metapolítica como:

- encuestas electorales;

- especulación sobre los pactos posteriores a los comicios;

- valoraciones respecto a las herramientas de campaña (vídeos o eslóganes);

- desarrollo de la campaña (si es aburrida, crispada, en clave local o imbuida por el debate soberanista);

- cuestiones conflictivas o espectacularizadas, como la expulsión violenta de miembros de la PAH (Plataforma de Afectados por la Hipoteca) en actos electorales del PP, la prohibición de colgar banderas independentistas en los ayuntamientos durante la campaña electoral o la militancia política de miembros de la iglesia catalana.

Las propuestas concretas de los partidos sólo se enumeran de forma sumaria en algún momento de todas las tertulias, pero la discusión a fondo de las propuestas sólo aparece en 4 de las 29 tertulias analizadas. Mayoritariamente

sino más bien a un recurso para polemizar y espectacularizar el espacio. La polarización ideológica y enfrentada, en cambio, sólo se produce en posiciones dicotómicas en torno al proceso independentista catalán y las estrategias electorales de los nuevos partidos de izquierdas.

En el resto de temas la interacción es más relajada y fluida, sin tantas interrupciones o subidas de intensidad en el discurso.

También hay que señalar la poca presencia de argumentación y su escasa calidad. Los tertulianos se limitan a exponer opiniones personales sin argumentos y cuando éstos aparecen, resultan poco elaborados y nada diversos. Se pronuncian sobre todo argumentos morales, legales o basados en ejemplos, mientras que abundan las generalizaciones, experiencias personales, especulaciones y expresión de intuiciones, como por ejemplo la opinión de la tertuliana Gemma Galdón el dia 11 de mayo en La rambla de BTV: "Lo que pide la gente en España, Cataluña y Barcelona es que cambien las cosas".

\section{Se pronuncian sobre todo argumentos morales, legales o basados en ejemplos y abundan las generalizaciones, expe- riencias personales, especulaciones y la expresión de intuiciones}

La emocionalidad prima ante las razones. En ocasiones incluso los tertulianos se autocitan, dan como referencia opiniones versadas anteriormente o en otros medios, demostrando así una conciencia de opinion makers. Es el caso entre otros de Joan B. Culla, tertuliano de Els matins de TV3, que cita sus propios artículos en prensa (19/05/2015). La preponderancia de la opinión personal no razonada viene marcada por el perfil de tertulianos, mayormente generalistas. Cabe destacar que en algunos casos se dan argumentos contextuales con datos concretos extraídos de fuentes formales como encuestas o comunicados institucionales, pero no es una tendencia mayoritaria (4 tertulias de las 29 analizadas). Esto confiere a las tertulias poca concreción y las 
convierte en un terreno abonado para el espectáculo banal.

En tanto que la metapolítica como contenido es el rasgo principal de todas las tertulias analizadas, los resultados confirman la existencia de la strategy coverage de Capella y Jamieson (1997). Encontramos una simplificación narrativa y un lenguaje en términos de dicotomía a la hora de explicar la realidad y de definir a los actores políticos: la vieja y la nueva política, el cambio y el status quo, la situación escocesa, la realidad de los pueblos y las ciudades, los políticos buenos y los malos.

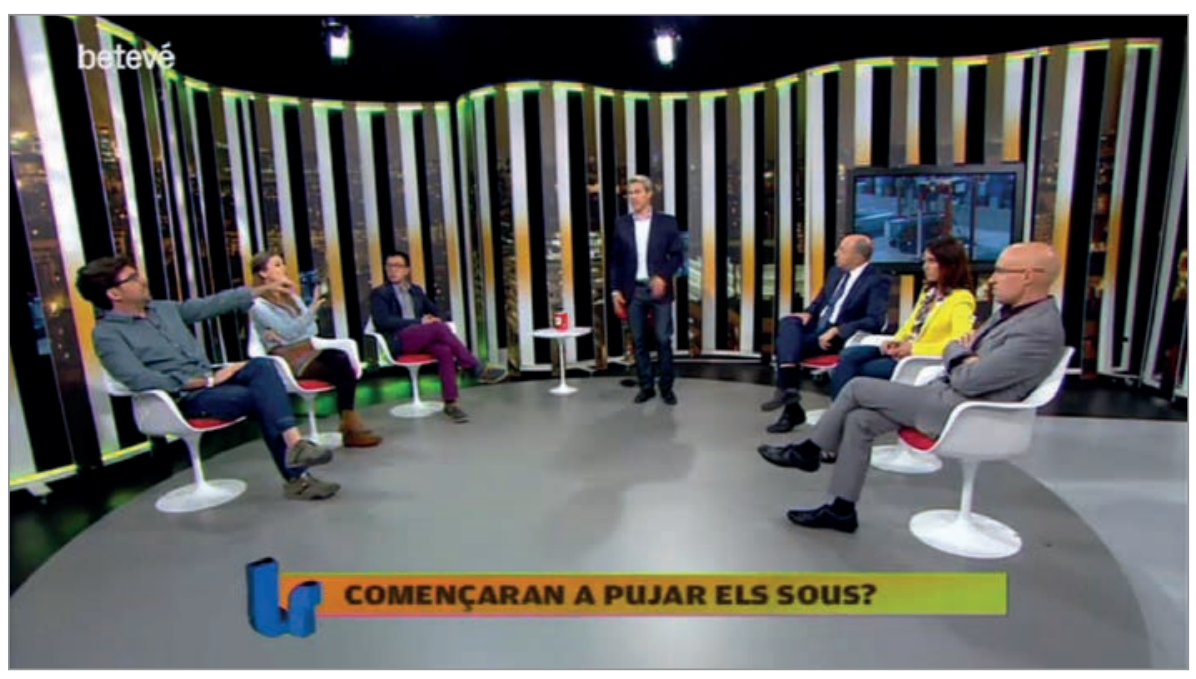

Tertulia La rambla, de la cadena local pública Barcelona Televisió (BTV)
Otra de las características de la strategy coverage implica la utilización de léxico bélico, que lleva a interpretar algunos de los temas relacionados con los comicios como una batalla, como se ejemplifica cuando se hace referencia al contexto político catalán y su influencia en las posiciones ideológicas en campaña:

"El enfrentamiento entre el Estado y Cataluña es una batalla impresentable" (Joan Manel Tresserres, Els matins de TV3, 15/05/2015).

"Es una campaña de guerracivilismo, de blanco y negro" (Pere Martí, 8 al dia, 12/05/2015).

Las referencias a las encuestas electorales están impregnadas de este tipo de léxico, que también se utiliza en las interpretaciones de las elecciones municipales como el primer "round" de los comicios del 27 de septiembre (El matins de TV3, 18/05/2015), convirtiendo la campaña electoral en un eslabón "estratégico" para las formaciones políticas. Curiosamente, los propios tertulianos que realizan este tipo de cobertura acusan a los partidos políticos de "realizar una campaña sin contenido, sólo a base de acciones estratégicas y propuestas tópicas y superficiales" (Joan-Josep Queralt, 8 al dia, 22/05/15).

La personalización se presenta tanto en las referencias a los políticos como a los tertulianos y en los presentadores. Siguiendo con la definición de Van-Santen y Van-Zoonen (2010), se remarcan cuestiones de la vida privada de los políticos, sus habilidades y sus opiniones, sobre todo en el caso de las televisiones privadas. Los éxitos y los fracasos se personalizan en los candidatos, no se atribuyen a los partidos o los gobiernos. Los líderes políticos más destacados en esta tendencia a la personalización son sobre todo Ada Colau y Artur Mas.

En algunos casos, se descalifica y ridiculiza a los políticos, sobre todo a Ada Colau, de quien se afirman cosas como:

"hace milagros, como ser activista y cobrar 1.900 euros del Ayuntamiento que califica de mafioso" (Bernat de Déu, La rambla, BTV, 15/05/15),

Mientras, otros candidatos pasan prácticamente desapercibidos. Esto se produce de forma clara en el caso de la televi- sión privada analizada, pero también en menor grado en el resto de la muestra.

Las tertulias tienen un tono más tranquilo en la televisión pública, y resultan algo más exaltadas en el caso de $8 T V$ i BTV. En cuanto al lenguaje no verbal, la gesticulación acostumbra a ser explicativa y, sólo en algunos casos, más expresiva. En los programas de BTV son frecuentes las interrupciones y los parlamentos desordenados y al mismo tiempo, hasta el punto que en alguna ocasión el moderador pide que se bajen los micrófonos. La realización más espectacularizada es la de La rambla, que podría responder al hecho de que es la única tertulia analizada que se emite en una franja nocturna de prime-time televisivo, un hecho que, como indican Gómez-Aguilar, Paniagua-Rojano y Farias-Batlle (2015) en su análisis sobre la tertulia La sexta noche y la actividad en las redes sociales, responde a una estrategia de máxima difusión del espacio, como también indican otros estudios sobre la interacción de programas televisivos y sus audiencias sociales (Halpern; Quintas-Froufe; Fernández-Medina, 2016).

\section{Las tertulias tienen un tono más tranqui- lo en la televisión pública, y más exalta- do en 8TV i BTV}

Mientras en la televisión pública catalana no se enfatiza la confrontación ni se detectan frecuentes movimientos de cámara, en $8 T V$ se subraya el conflicto a través de la pantalla partida en dos e incluso en tres partes y en BTV hallamos numerosos rasgos del infoentretenimiento:

- el programa empieza con los tertulianos entrando por el plató en charla informal, con planos que enfocan los pies; - son frecuentes los movimientos de la cámara grúa, los picados y contrapicados y la pantalla partida en dos;

- en los vídeos que introducen los temas se utiliza música y un efecto de marco desenfocado;

- la disposición del plató es en semicírculo, con el moderador en el medio y tertulianos a cada uno de los lados. En esto también hay un cambio importante entre TV3 y las otras dos cadenas televisivas. En TV3, el moderador in- 
troduce los temas de forma neutra, pero en $8 T V$ y en BTV, Josep Cuní y Daniel Domenjó actúan según las características propias que León-Gross y Gómez-Calderón (2011) dan a los moderadores de las tertulias: actúan como elementos identificativos de la cadena y del espacio, son reconocidos como autoridad por los tertulianos y marcan no tanto los temas, prácticamente iguales en todas las tertulias, sino sobre todo los encuadres.

\section{Conclusiones}

Los rasgos del género "infoentretenimiento" están presentes en las tertulias televisivas durante la campaña electoral y se perciben tanto en los discursos como en las formas y el lenguaje televisivo, aunque parece haber una diferencia clara entre las cadenas: Televisió de Catalunya mantiene un perfil menos espectacularizado, mientras que en $8 T V$ está más marcado.

Mención especial merece el programa La rambla, con diferencia la tertulia política más espectacularizada de las analizadas, pero también el espacio, por ámbito de cobertura, que más temas de los programas electorales de los partidos políticos trata, aunque no en profundidad ni con la presencia de expertos.

\section{Los partidos políticos y los periodistas} reclaman espacios más dúctiles y libres para explicar mejor sus propuestas y sus investigaciones

La figura predominante es el denominado "tertuliano-estreIla", que tiene un perfil generalista, de formación periodística. Un $80 \%$ de los tertulianos responden a este perfil. El resto -economistas, abogados, académicos o ex-políticos en su mayoría- no son distribuidos en la programación según los temas a tratar como expertos o especialistas, sino que su presencia se produce por su perfil público. En este caso, y obviando la especialidad de su ámbito profesional, la figura del experto actúa también de forma espectacularizada y generalista, especialmente en la construcción argumental. Ésta se basa fundamentalmente en lugares comunes y anécdotas personales, mientas que en pocos casos se aporta argumentación con datos y de contexto. Esto, como indican Castelló y Montagut, (2011) puede deberse a las rutinas productivas de estos espacios, dado que cubren espacios programáticos amplios y de periodicidad diaria, no tienen tiempo de producir tertulianos ad hoc para cada programa y establecen una pauta fija de programación de opinadores donde el rol generalista permite más comodidad en las rutinas productivas.

Este rol generalista conlleva, en el caso de los contenidos políticos, pautas de tratamiento común-strategy coverage, simplificación argumental dicotómica, personificación de los actores políticos, etc.-, además de la búsqueda de temas que permitan desplegar esas pautas como los temas conflictivos o la metapolítica. De hecho, la metapolítica se consolida como un recurso transversal para tratar incluso otros temas de mayor calado como la crisis económica o el modelo turístico de la ciudad desde un esquema reduccionista. Cuando Cappella y Jamieson (1997) establecen el concepto de la "espiral del cinismo" en la relación entre políticos y periodistas, muchas de las razones que dan unos y otros para generar desconfianza mutua están relacionadas precisamente con un tratamiento de la información y la opinión política simplificada y banal, tanto por parte de los periodistas como por parte del control de los partidos sobre la información (Castelló; Montagut, 2011).

El análisis efectuado permite confirmar que el infoentretenimiento permea en muchos espacios mediáticos, no sólo los informativos o los latenights, sino también magazines de actualidad. La preponderancia de la metapolítica sobre las propuestas; la entronización de la anécdota en detrimento de la noticia relevante explicada en toda su complejidad, en el sentido de relato que describe Salmon (2008); la sensación de carrera de caballos que indica Maarek (1997) o la construcción dramática de los actores políticos que señala Van-Zoonen (2005), indican que estamos ante un tipo de espectáculo que lejos de empoderar, como señala Bennett (1992), empobrece.

En suma, los partidos políticos y los periodistas, que reclaman espacios más dúctiles y libres para explicar mejor sus propuestas y sus investigaciones, pierden una oportunidad excelente para ello con la orientación de las tertulias hacia el espectáculo banal. Estamos de acuerdo con Pont-Sorribes et al. (2016) cuando apuntaban a la necesidad de mejorar el perfil de los tertulianos, potenciando no sólo la figura del experto, sino evitando una pluralidad espectacularizada o indirectamente politizada y apostar por una pluralidad de perfiles profesionales, ideológicos, generacionales y de género.

Por último, queda por explorar si la espectacularización en las tertulias políticas televisivas tiene una incidencia negativa -desafección política- o positiva -ciudadanía informadasobre la formación de la opinión pública. 


\section{Bibliografía}

Abejón-Mendoza, Paloma (2013). “Estado del periodismo de opinión en España: polarización, género y emolumentos". ZER, v. 18, n. 34, pp. 93-109.

http://www.ehu.eus/zer/hemeroteca/pdfs/zer34-05-abejon.pdf

Aira, Toni (2009). Els spin doctors. Com mouen els fils els assessors dels líders polítics. Barcelona: L'arquer. ISBN: 974 8497888066

Altarriba-Piguillem, Laia (2013). Informe Media.cat. Qui són els que ens formen l'opinió?

http://www.media.cat/wp-content/uploads/2013/03/ Informe_Mediacat_Opinadors.pdf

Bennett, Lance (1992). "White noise: The perils of mass mediated democracy". Communication monographs, n. 59, n. 4, pp. 401-406.

https://goo.gl/2Umfca

https://doi.org/10.1080/03637759209376281

Berrocal-Gonzalo, Salomé; Redondo-García, Marta; Martín-Jiménez, Virginia; Campos-Domínguez, Eva (2014): “La presencia del infoentretenimiento en los canales generalistas de la TDT española". Revista latina de comunicación social, v. 69, pp. 85-103.

https://doi.org/10.4185/RLCS-2014-1002

Blasco-Duatis, Marc (2013). Propuesta metodológica para el análisis de la tertulia política en televisión. Universidad de Coimbra. Tesis doctoral

https://mduatis.files.wordpress.com/2013/07/tese_blasco_ duatis_fluc2013.pdf

Brants, Kees (1998). "Who's afraid of infotainment?". European journal of communication, v. 13, n. 3, pp. 315-336. https://www.researchgate.net/publication/249720368_ Who's_Afraid_of_Infotainment

https://doi.org/10.1177/0267323198013003002

Capella, Joseph; Jamieson, Katheleen (1997). Spiral of cynicism: The press and the public good. New York: Oxford University Press. ISBN: 0195090632

Carrillo, Nereida (2012). El xou nostrat. L'infoentreteniment en el contingut i la recepció del periodisme electoral a Catalunya en els casos de les eleccions de 2008 i 2010. Tesi doctoral. Universitat Autònoma de Barcelona.

http://www.tesisenred.net/handle/10803/107823

Carrillo, Nereida (2013). El periodismo volátil. ¿Cómo atrapar la información política que se nos escapa?. Barcelona: Editorial UOC. ISBN: 9788490299814

Casero-Ripollés, Andreu (2008). “Modelos de relación entre periodistas y políticos: La perspectiva de la negociación constante". Estudios sobre el mensaje periodístico, n. 14, pp. 111-128.

http://repositori.uji.es/xmlui/handle/10234/9053

Castelló, Enric (ed.) (2012). La mediatización del conflicto político: discursos y narrativas en el contexto español. Barcelona: Laertes. ISBN: 9788475848792

Castelló, Enric; Montagut, Marta (2012). “Journalists, reframing and party public relations consultants. Strategies in morning talk radio". Journalism studies, v. 12 , n. 4, pp. 506-521.

https://doi.org/10.1080/1461670X.2010.530969

Cebrian, Elena; Berrocal-Gonzalo, Salomé (2013). “La irrupción del 'infoentretenimiento' en la comunicación política española. Una propuesta para la sistematización de sus formatos televisivos". En: Sanders, Karen; Canel, María-José; Capdevila, Arantxa; Gurrionero, Mario (coords.). Estudios de comunicación política. Libro del año 2012. ACOP. Madrid: Tecnos, pp. 260-275. ISBN: 9788430955305

Debord, Guy (1992). La societé du spectacle. Paris: Éditions Gallimard. ISBN: 2070394433

Edelman, Murray (1988). Constructing the political spectacle. Chicago: The University of Chicago Press. ISBN: 0 226183998

Ferré-Pavía, Carme; Sintes-Olivella, Marçal; Gayà-Morlà, Catalina (2012). “¿Piensan lo mismo ciudadanos y partidos sobre la sátira política? La diferente percepción en el caso de Polònia". Estudios sobre el mensaje periodístico, v. 19, n. 1 pp. 383-402

https://doi.org/10.5209/rev_ESMP.2013.v19.n1.42528

Früh, Werner; Wirth, Werner (1997). "Positives und negatives Infotainment. Zur Rezeption unterhaltsam aufbereiteter TV-Information". En: Haller, Michael; Bentele, Günter. Aktuelle entstehung von öffentlichkeit. Akteure-strukturenveränderungen. Konstanz: UVK, pp. 367-381.

Gómez-Aguilar, Marisol; Paniagua-Rojano, Franciso-Javier; Farias-Batlle, Pedro (2015). "Comportamiento de la audiencia de televisión en las redes sociales. Una aproximación al perfil y programas más comentados". Revista latina de comunicación social, n. 70, pp. 539-551.

https://doi.org/10.4185/RLCS-2015-1058

Gutiérrez-García, María; Huertas-Bailén, Amparo (2004). "Radio: España, programación radial para todo público". Ensayos. Ciespal n. 86, pp. 46-51.

http://hdl.handle.net/10469/10549

Halpern, Daniel; Quintas-Froufe, Natalia; Fernández-Medina, Francisco (2016). "Interacciones entre la televisión y su audiencia social: hacia una conceptualización comunicacional". El profesional de la información, v. 25, n. 3, pp. 367-375. https://doi.org/10.3145/epi.2016.may.06

Hallin, Daniel; Mancini, Paolo (2004). Comparing media systems. Three models of media and politics. New York: Cambridge University Press. ISBN: 0521835356 https://goo.gl/GRXBJE

Jones, Jeffrey (2005). Entertaining politics: New political television and civic culture. Lanham: Rowman \& Littlefield. ISBN: 9780742530881

Kellner, Douglas (2003). Media spectacle. Londres: Routledge. ISBN: 9780415268288

León-Gross, Teodoro; Gómez-Calderón, Bernardo (2011). "La tertulia en España: medios públicos, la última frontera de la pluralidad". Estudios sobre el mensaje periodístico, v. 17, n. 1, pp. 67-80.

https://doi.org/10.5209/rev_ESMP.2011.v17.n1.4 
Maarek, Philippe (1997). Marketing político y comunicación. Barcelona: Paidós. ISBN: 9788449304514

O’Donnel, Hugh (2007). Noticias y ciudadanía. El telespectador, el poder y el debate público. Madrid: Ediciones de la Torre. ISBN: 9788479603762

Ortells-Badenes, Sara (2015). “Los magazines de actualidad basados en el infoentretenimiento: nuevos rasgos del lenguaje audiovisual en el periodismo televisivo". Signo y pensamiento, v. 34, n. 66, pp. 44-61

https://doi.org/10.11144/Javeriana.syp34-66.mabi

Pont-Sorribes, Carles; Alonso, Felipe; Gili, Ricard; Mercader, Albert (2016). L'articulació del discurs i el clima d'opinió de la Tardor Catalana a les tertúlies de Televisió de CataIunya i Catalunya Ràdio. Informe CAC 2016. IX Convocatòria dels ajuts a la recerca sobre comunicació audiovisual. Barcelona: Consell Audiovisual de Catalunya.

https://www.cac.cat/components/generic/file/download. jsp?idFichero $=30942$

Postman, Neil (1990). Divertim-nos fins a morir. El discurs públic a l'època del show-business. Barcelona: Llibres de l'índex. ISBN: 9788487561061

Rico-Jerez, Marta (2014). “La aplicación de los géneros informativos e interpretativos en las tertulias políticas del
Grupo Intereconomía". Estudios sobre el mensaje periodístico, v. 20, n. 2, pp. 873-884.

https://doi.org/10.5209/rev_ESMP.2014.v20.n2.47060

Salmon, Christian (2008). Storytelling: la máquina de fabricar historias y formatear mentes. Barcelona: ediciones Península. ISBN: 9788499424989

Sánchez-Serrano, Chelo (2006). "Las tertulias políticas de la radio española. Un ejercicio de comunicación política mejorable". Comunicación y pluralismo, v. 0, pp. 141-152.

https://goo.gl/ZqgxCQ

Toral-Madariaga, Gotzon (1998). Tertulias, mentideros y programas de radio. Bilbao: Alberdania. ISBN: 97884 88669643

Thussu, Daya (2007). News as entertainment. The rise of global infotainment. Londres: SAGE. ISBN: 9780761968788

Van-Santen, Rosa; Van-Zoonen, Liesbet (2010). "The personal in political television biographies". Biography, v. 33, n. 1, pp. 46-67.

https://goo.gl/AK4jLi

https://doi.org/10.1353/bio.0.0157

Van-Zoonen, Liesbet (2005). Entertaining the citizen. When politics and popular culture converge. Lanham: Rowman \& Littlefield Publishers Inc. ISBN: 9780742529076

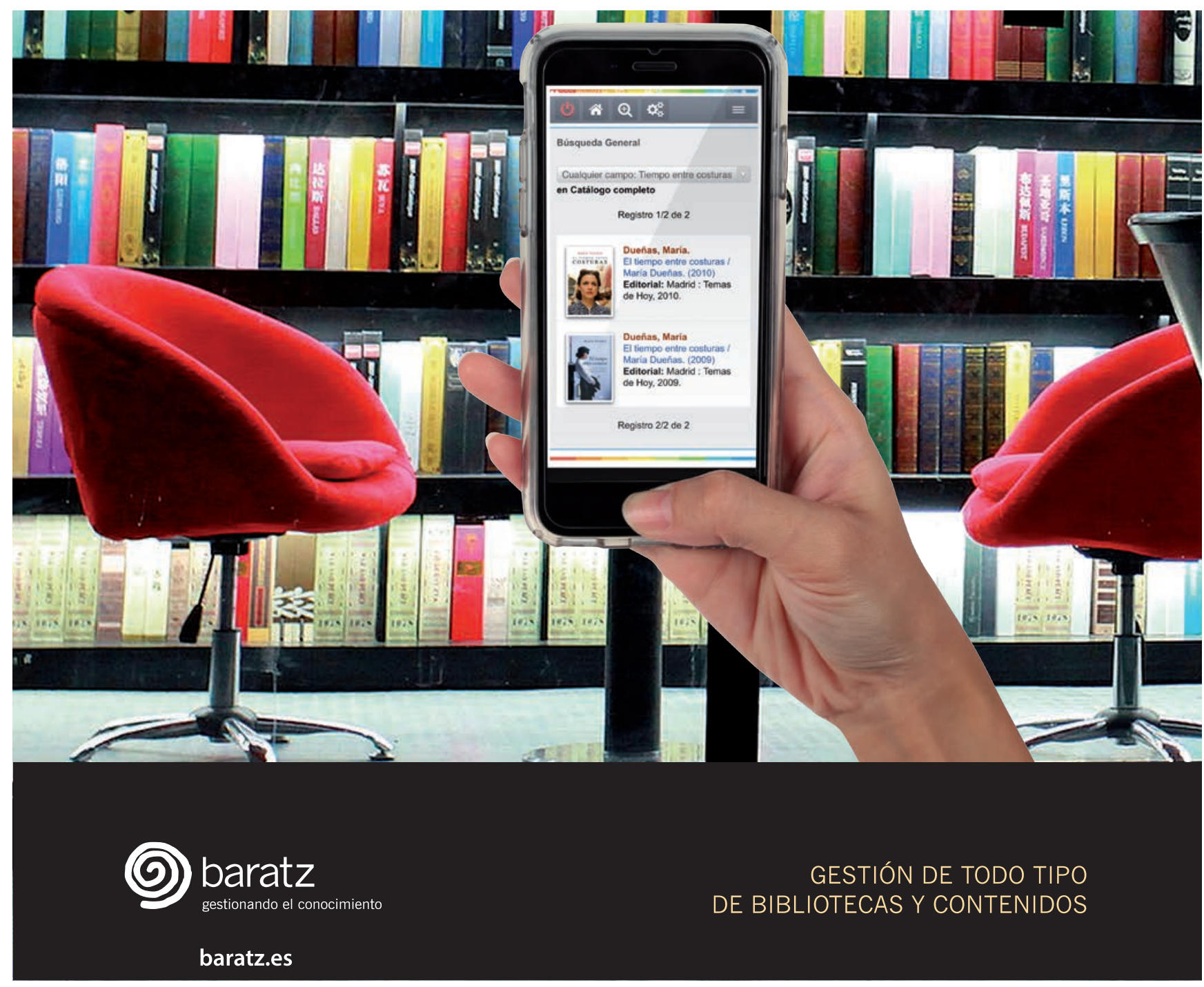

